\title{
Prevalencia del síndrome metabólico, gravedad clínica y calidad de vida en pacientes con psoriasis del Hospital Universitario de La Samaritana
}

Prevalence of metabolic syndrome, clinical severity and quality of life in patients with psoriasis at the Hospital Universitario de La Samaritana.

\section{Rolando Rojas ${ }^{1}$, Héctor Castellanos ${ }^{2}$, Elkin Peñaranda ${ }^{3}$}

1. Médico dermatólogo, Universidad Nacional de Colombia, Bogotá, D.C., Colombia.

2. Médico dermatólogo; profesor asociado de Dermatología, Universidad Nacional de Colombia, Bogotá, D.C., Colombia.

3. Médico dermatólogo oncólogo; coordinador, Servicio de Dermatología, Hospital Universitario de La Samaritana, Bogotá, D.C., Colombia.

\section{Resumen}

Introducción. Actualmente la psoriasis se reconoce como una enfermedad sistémica y con ello han surgido trabajos que la relacionan con otras entidades, entre ellas con el síndrome metabólico, el cual se asocia con un mayor riesgo de desarrollar eventos cardiovasculares. Las implicaciones sobre la calidad de vida de los individuos que padecen la enfermedad abarcan no solamente los aspectos físicos, sino también los psicológicos, sociales, sexuales y ocupacionales.

Materiales y métodos. Se hizo un estudio descriptivo y prospectivo, en el que se incluyeron pacientes con diagnóstico de psoriasis, a quienes se les calculó el Psoriasis Area Severity Index (PASI) y el Dermatology Life Quality Index (DLQI), y se les investigó la presencia de los componentes del síndrome metabólico con base en los criterios de la International Diabetes Federation (IDF) para poblaciones suramericanas.

Resultados. Se incluyeron 52 pacientes -26 hombres y 26 mujeres- cuyo rango de edad se ubicó, principalmente, entre los 41 y los 64 años (69,2 \%); la psoriasis fue leve en la mayoría, con el 67,3\%. El 84,6 \% presentó compromiso de su calidad de vida. El síndrome metabólico estuvo presente en el 59,6 \% y, la artritis psoriásica, en el 19,2\%.

Conclusión. Los resultados de este estudio se convierten en una base referencial del comportamiento de la psoriasis en una población colombiana, sobre el que actualmente se cuenta con poca información.

PALABRAS CLAVE: psoriasis, calidad de vida, síndrome metabólico.

\section{Summary}

Introduction: Psoriasis is recognized as a systemic disease, and some studies have associated this disease with other entities, such as the metabolic syndrome, which has a significant association with an increased risk of cardiovascular events. Its impact on patients' lives is not only related with the physical, but also with the other components of quality of life, including psychological, social, sexual and occupational elements.

Materials and methods: A prospective and descriptive study that included pa-

\section{Correspondencia:}

Rolando Rojas

Email:

dermatologia84@yahoo.com

Recibido: 10 de abril de 2013. Aceptado: 21 de junio de 2013.

No se reportan conflictos de intereses. 
tients with psoriasis was made; Psoriasis Area and Severity Index (PASI) and Dermatology Life Quality Index (DLQI) were measured; and the presence of metabolic syndrome components based on the criteria of the International Diabetes Federation (IDF) to South American populations was established.

Results: We included 52 patients, 26 men and 26 women, whose age range was located mainly between 41 and 64 years old $(69.2 \%)$, psoriasis was mostly mild in $67.3 \%$, quality of life was affected in $84.6 \%$ of patients. Metabolic syndrome was present in $59.6 \%$ and psoriatic arthritis in $19.2 \%$.

Conclusion: The results of this study will become a baseline of the behavior of psoriasis in a Colombian population, because nowadays there is little information about this topic.

KEYWORDS: Psoriasis, quality of life, metabolic syndrome.

\section{Introducción}

Los grandes avances en el conocimiento de la psoriasis han permitido revelar aspectos trascendentales de su epidemiología, inmunología, biología molecular e inmunogenética pero, a pesar de ello, hasta el momento no existe una cura definitiva y todavía queda mucho por conocer sobre esta entidad, relaciones por establecer y preguntas por resolver.

Dentro del marco actual de su entendimiento, la psoriasis se reconoce como una enfermedad sistémica, con un compromiso que va más allá del cutáneo; con ello han ido surgiendo trabajos que la relacionan con otras entidades con las que previamente pudiese haberse considerado que su vínculo no tendría cabida, por ejemplo, con el síndrome metabólico, cuya presencia predispone a los pacientes afectados a un mayor riesgo de desarrollo de eventos cardiovasculares, con todas las consecuencias que esto representa desde el punto de vista de la morbimortalidad.

Es claro que las implicaciones sobre la vida de los individuos con psoriasis no solamente tienen que ver con los aspectos físicos de su enfermedad, sino que, además, se pueden ver afectados en todos los otros componentes de la calidad de vida, que incluyen elementos psicológicos, sociales, sexuales y ocupacionales.

En Latinoamérica, y específicamente en Colombia, existen pocos trabajos relacionados con la caracterización epidemiológica de la psoriasis; igualmente, son aislados los estudios en relación con el síndrome metabólico y la calidad de vida de los individuos afectados ${ }^{1-7}$.

Un panorama como este motiva a que se realicen estudios dirigidos a fortalecer la información disponible frente a las características de la psoriasis en la pobla- ción colombiana, que permitan compararla con poblaciones de otros lugares.

El Hospital de La Samaritana es una de las instituciones prestadoras de servicios de salud más importantes del país, centro de referencia de pacientes dermatológicos de la ciudad de Bogotá y de los municipios del departamento de Cundinamarca, lo que lo convirtió en un espacio apto para llevar a cabo este trabajo, cuyos aportes adquieren de entrada un valor importante frente al conocimiento local de la enfermedad.

\section{Materiales y métodos}

Se llevó a cabo un estudio observacional descriptivo, prospectivo, con un muestreo por conveniencia, en el cual se incluyeron todos los pacientes mayores de 18 años con diagnóstico de psoriasis en placas, según los puntos de vista clínico e histopatológico, valorados por un dermatólogo integrante de la Clínica de Psoriasis del Hospital de La Samaritana de Bogotá, entre junio de 2010 y junio de 2012, que aceptaron participar de manera voluntaria, previa firma del consentimiento informado.

Del estudio se excluyeron los pacientes menores de edad o con alteraciones mentales o con alguna enfermedad concomitante que limitara la capacidad de diligenciar los instrumentos de medición planteados en este estudio, y los pacientes en quienes existieran dudas respecto al tipo de enfermedad dermatológica o con voluntad expresa de no participar en el estudio.

A todos los pacientes se les diligenció un formato de historia clínica que incluía la evaluación de características sociodemográficas, los antecedentes personales de hipertensión arterial, diabetes mellitus o dislipidemia, y si se encontraban o no en tratamiento para alguna de 


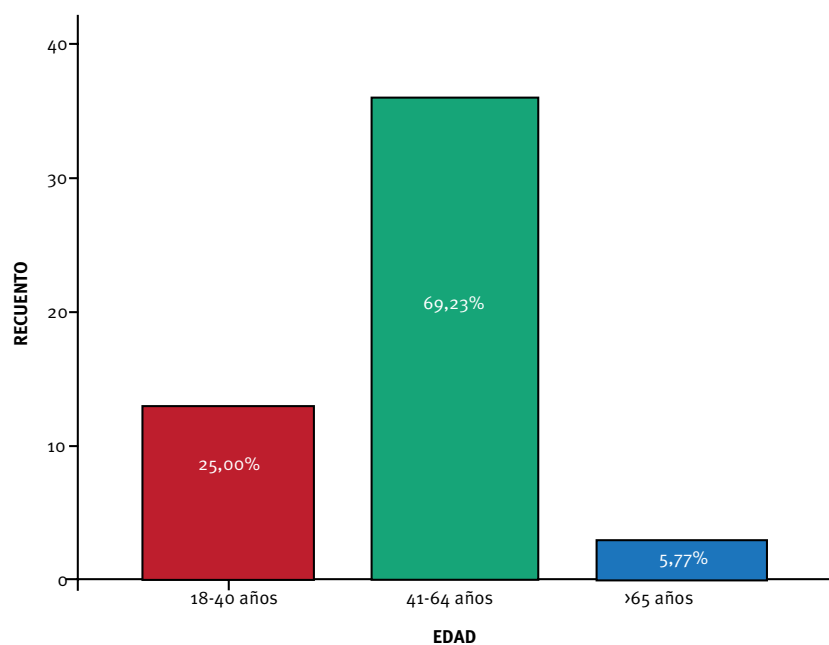

FIGURA 1. Distribución de los pacientes según los grupos etarios.

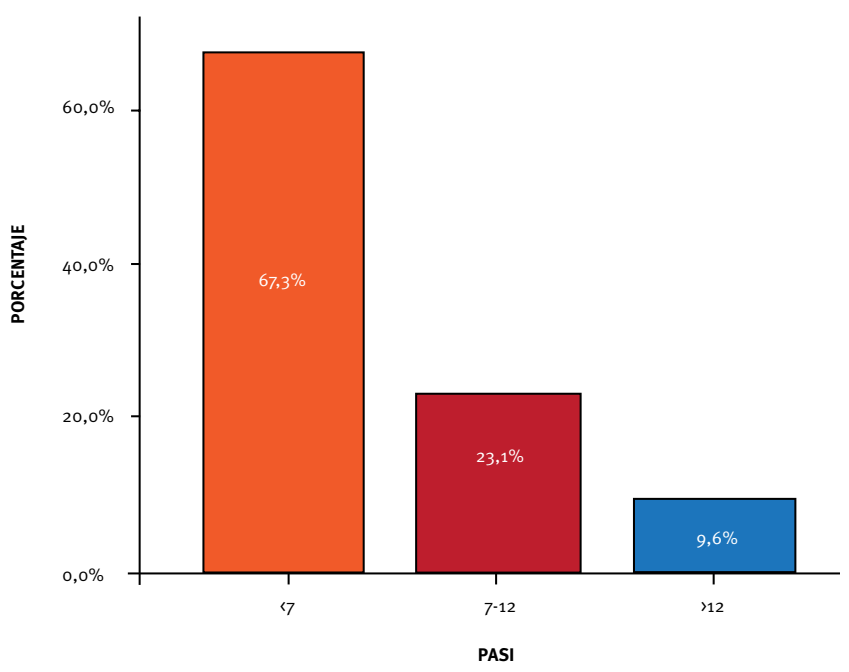

FIGURA 2. Gravedad clínica de la psoriasis, según el PASI. estas condiciones médicas. Se les calculó el Psoriasis Area Severity Index (PASI) para determinar la gravedad clínica de la psoriasis, según los puntos de corte establecidos, y el Dermatology Life Quality Index (DLQI) para evaluar el compromiso de la calidad de vida.

Además, se investigó la presencia de los componentes del síndrome metabólico con base en los criterios de la International Diabetes Federation (IDF) para poblaciones suramericanas. El síndrome metabólico se confirmó según la presencia de obesidad abdominal, establecida por un perímetro de la cintura en hombres igual o mayor de $90 \mathrm{~cm}$, y en mujeres, igual o mayor de $80 \mathrm{~cm}$, más dos de los siguientes criterios: glucosa venosa en ayunas igual o mayor de $100 \mathrm{mg} / \mathrm{dl}(5,6 \mathrm{mmol} / \mathrm{L})$, incluye diabetes; presión arterial mayor o igual de $130 / 85 \mathrm{~mm} \mathrm{Hg}$ o estar consumiendo medicamentos antihipertensivos; colesterol HDL menor de $40 \mathrm{mg} / \mathrm{dl}(1,03 \mathrm{mmol} / \mathrm{L})$ en hombres y menor de $50 \mathrm{mg} / \mathrm{dl}(1,29 \mathrm{mmol} / \mathrm{L})$ en mujeres, o estar tomando medicamentos hipolipemiantes; triglicéridos iguales o mayores de $150 \mathrm{mg} / \mathrm{dl}(1,7 \mathrm{mmol} / \mathrm{L})$ o estar en tratamiento con hipolipemiantes.

La artritis psoriásica se diagnosticó con base en los criterios CASPAR (Classification criteria for Psoriatic Arthritis), por parte de un reumatólogo integrante de la Clínica de Psoriasis.

Toda la información se almacenó en una base de datos construida en Excel ${ }^{\circledR}$ y los datos fueron analizados en el programa estadístico EpiInfo.

\section{Resultados}

Se incluyeron 52 pacientes, 26 hombres y 26 mujeres. En el momento del estudio, 36 pacientes $(69,2 \%)$ se encon- traban en el rango de edad entre los 41 y los 64 años seguido por 13 pacientes ( $25 \%$ ) entre los 18 y los 40 años y 3 pacientes $(5,8 \%)$ de 65 años o más (FIGURA 1).

Según el PASI y los puntos de corte establecidos en este estudio, el 67,3\% (35) de los pacientes tenía una psoriasis leve en el momento del examen (PASI $<7$ ), seguido del 23,1 \% (12) con psoriasis moderada (PASI entre 7 y 12) y del 9,6 \% (5) con psoriasis grave (PASI>12) (FIGURA 2).

La artritis psoriásica estaba presente en el 19,2 \% de los enfermos (10 pacientes). Se encontró que el 73,1 $\%$ (38 pacientes) presentaba obesidad abdominal; el $61,5 \%$, dislipidemia de tipo de hipertrigliceridemia (32 pacientes) y el $61,5 \%$, disminución del colesterol HDL (32 pacientes). En relación con la glucemia, el 36,5 \% (19) presentaba antecedentes de diabetes mellitus o alteración de sus niveles plasmáticos. El 61,5 \% (32 pacientes) tenía cifras elevadas de presión arterial o antecedentes de hipertensión arterial. El 59,6 \% (31 pacientes) cumplía los criterios para síndrome metabólico (FIGURA 3).

En el 84,6\% (44) de los casos se presentó compromiso de la calidad de vida; de manera leve (DLQI: 2-5) en el $25 \%$ (13), moderada (DLQI: 6-10) en el 26,9\% (14), grave (DLQI: 11-20) en el $25 \%$ (13) y muy grave (DLQI: 21-30) en el $7,7 \%$ (4) (FIGURA 4).

De los pacientes con enfermedad clínicamente leve (PASI $<7$ ), que fueron la mayoría, el 17,1 \% (6 pacientes) no presentaba compromiso de su calidad de vida, el 25,7 $\%$ (9) tenía un compromiso leve de su calidad de vida; el $22,9 \%$ (8), un compromiso moderado; el 25,7 \% (9), un compromiso grave, y el 8,6\% (3), un compromiso muy grave; entre estos mismos pacientes, el 62,9 \% (22) cumplió criterios para síndrome metabólico. 


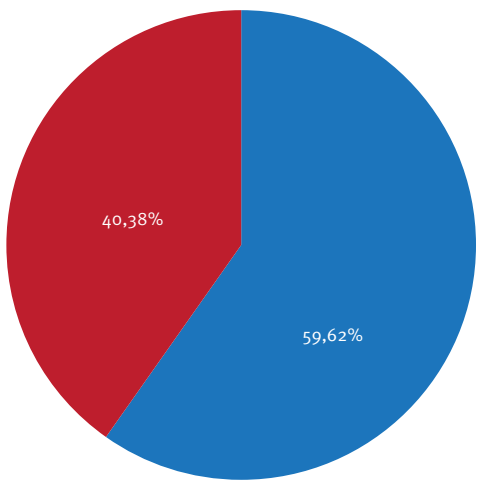

SÍNDROME METABÓLICO

$\mathrm{Si}$ No

FigurA 3. Prevalencia de síndrome metabólico en los pacientes con psoriasis.

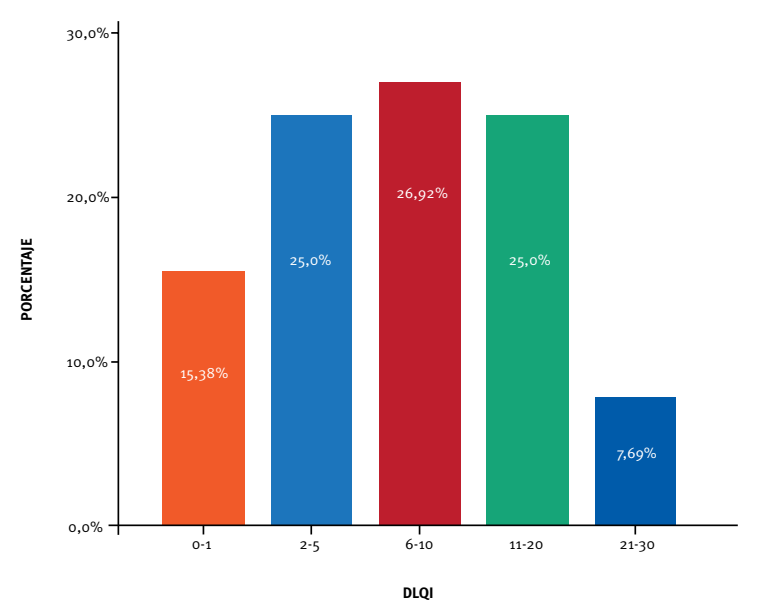

FigurA 4. Compromiso de la calidad de vida de los pacientes con psoriasis, según el DLQI.

\section{Discusión}

En este estudio descriptivo de 52 pacientes con psoriasis se encontró un porcentaje similar de afectación en hombres y mujeres, lo que se ajusta con lo referido en la literatura científica, en la cual se muestra que en esta enfermedad no existe predilección por ningún sexo en la edad adulta ${ }^{1,7-10}$.

En relación con la edad, el grupo etario entre los $41 \mathrm{y}$ los 64 años representó un alto porcentaje al momento de la realización del trabajo, lo cual coincide con lo descrito en otros estudios, en los cuales la edad promedio de los pacientes, generalmente, se ubica en la quinta década de la vida ${ }^{1,4,7,10}$.

La artritis psoriásica estuvo presente en el 19,2\% de los casos; los estudios reportan una prevalencia que va del 6 al $42 \%$ en los pacientes con psoriasis ${ }^{11-14}$.

De acuerdo con la literatura científica, el PASI es el sistema de medición de gravedad clínica más válido y reproducible en el manejo de un paciente adulto con psoriasis. Según el PASI, la psoriasis se puede clasificar como leve (PASI<7), moderada (PASI: 7-12) o grave $(\mathrm{PASI}>12)^{15-17}$. Afortunadamente, la mayoría de los pacientes tiene enfermedad clínicamente leve $\mathrm{e}^{18}$, lo que se corrobora en este estudio, en el cual se encontró que la enfermedad fue leve en el $67,3 \%$ de los casos.

Durante las dos últimas décadas, el síndrome metabólico se ha convertido en uno de los principales retos para la salud pública en todo el mundo. La psoriasis se ha relacionado con elevación de la mortalidad por enfermedades cardiovasculares y muchas investigaciones se han emprendido para establecer el vínculo entre esta enfermedad cutánea y el aumento del riesgo cardiovascular ${ }^{18-24}$. Sin embargo, en nuestras poblaciones, un tanto heterogéneas, se sabe poco de esta presentación, su prevalencia y sus asociaciones.

En este estudio del Hospital de La Samaritana, con base en los criterios de la IDF para poblaciones suramericanas ${ }^{25,26}$, el 59,6 \% de los pacientes presentó síndrome metabólico. Se resalta, además, que la obesidad, la dislipidemia y la hipertensión arterial, estuvieron presentes en más de la mitad de los pacientes. Se encontró que el $73,1 \%$ presentó obesidad abdominal y, el 61,5\%, dislipidemia (tanto hipertrigliceridemia como alteración de las proteínas de alta densidad) e, igualmente, hipertensión arterial; en cambio, aunque para nada despreciable, el $36,5 \%$ presentó antecedentes de diabetes mellitus o alteración de los niveles plasmáticos de glucemia al momento del estudio.

Algunos autores han sugerido que la alta incidencia de síndrome metabólico en los individuos con psoriasis puede explicarse en parte por la inflamación crónica presente en la enfermedad. La psoriasis es un estado inflamatorio crónico y se ha sugerido que la inflamación crónica hace parte del síndrome metabólico. La inflamación crónica de la psoriasis tiene un efecto desfavorable en el perfil de riesgo cardiovascular. Múltiples factores de riesgo cardiovascular parecen ser influenciados: la presión arterial, el estrés oxidativo, la dislipidemia, la disfunción endotelial, los niveles de homocisteína y la adhesión plaquetaria ${ }^{27}$.

En este estudio, a pesar de que desde el punto de vista clínico la gravedad de la enfermedad fue leve en su mayoría, un alto porcentaje de pacientes presentó altera- 
ciones de su calidad de vida, lo que se ajusta a lo que han registrado otros trabajos, en los cuales no se ha encontrado una relación estadísticamente significativa entre la gravedad clínica de la psoriasis y la afectación de la calidad de vida ${ }^{6}$; de todas maneras, otros estudios reportan que, por supuesto, tener un PASI elevado tiene un impacto negativo en la calidad de vida ${ }^{16}$, lo que demuestra que no existe necesariamente una correlación definida entre estas dos variables de la enfermedad.

Existen datos que sugieren que el estigma social, los altos niveles de estrés, las limitaciones físicas, la depresión, los problemas de empleo y otros factores psicosociales experimentados por los pacientes con psoriasis, no son siempre proporcionales o predichos por otras medidas de la gravedad de la enfermedad, tales como el área de superficie corporal comprometida o la intensidad de las placas, lo cual ha hecho incluir medidas de morbilidad psicosocial cuando se evalúa la gravedad de la psoriasis, debido al papel sustancial que la carga psicosocial desempeña en la percepción de la complejidad de la enfermedad experimentada por el paciente, e igualmente, en la calidad de vida y en el curso de la enfermedad ${ }^{28-30}$.

En conclusión, los resultados obtenidos a partir de este estudio coinciden en gran medida con otros reportados en la literatura médica y contribuyen a fortalecer la información disponible frente al conocimiento de la psoriasis en nuestro medio.

Por sus hallazgos y los de otros estudios similares, se sugiere que a los pacientes con psoriasis se les haga tamización de los factores de riesgo asociados al síndrome metabólico, con el objetivo de dirigir intervenciones tempranas que tengan un impacto positivo frente a su potencial de morbimortalidad, todo bajo un enfoque multidisciplinario, incentivándolos, además, a modificar su estilo de vida. Asimismo, se recomienda valorar la calidad de vida de los pacientes, como factor determinante de su evaluación integral.

\section{Referencias}

1. Trujillo IA, Díaz-García MA, Torres-Gemeil O, Torres-Barbosa F, Falcón-Lincheta L, Pérez-Hernández M. Psoriasis vulgar. Estudio descriptivo de 200 pacientes. Rev Cubana Med. 2002;41:12-5.

2. González C, Castro LA, De La Cruz G, Arenas CM, Beltrán A, Santos AM. Caracterización epidemiológica de la psoriasis en el Hospital Militar Central. Rev Asoc Colomb Dermatol. 2009;17:11-7.

3. Vélez N, Bohórquez L, Corrales MF, Orrego S, Jiménez SB, Zuluaga $\mathrm{A}$, et al. Caracterización de los pacientes con psoriasis en tratamiento con fototerapia. Rev Asoc Colomb Dermatol. 2011;19:100-6.

4. Ramos E, Gioppo J, Miret M, Guzmán Y, Endara M. Factores de riesgo cardiovascular en pacientes con psoriasis. Dermatol Venez. 2008;46:11-8.
5. Carbo E, Leban V, Federico D, Williner ME, Díaz MG, Guardati $\mathrm{MV}$, et al. Psoriasis y síndrome metabólico: estudio restrospectivo sobre 22 casos. Rev Argent Dermatol. 2010;91:1-15.

6. Mussallam S, Meza B, Elescano I. Síndrome metabólico como factor asociado a psoriasis en la consulta dermatológica. Centro Médico Naval “CMST”, 2009. Folia Dermatol Perú. 2010;21:67-70.

7. Acosta-Medina D, Alfonso-Trujillo I, Toledo-García M. Calidad de vida, actividad y severidad de la psoriasis en pacientes de un policlínico de La Habana-Cuba. Enero-julio 2008. Dermatol Perú. 2009;19:44-7.

8. Elder JT, Bruce AT, Gudjonsson JE, Johnston A, Stuart PE, Tejasvi $\mathrm{T}$, et al. Molecular dissection of psoriasis: Integrating genetics and biology. J Invest Dermatol. 2010;130:1213-26.

9. Gudjonsson JE, Elder JT. Psoriasis: Epidemiology. Clin Dermatol. 2007;25:535-46.

10. Cohen AD, Gilutz H, Henkin Y, Zahger D, Shapiro J, Bonneh DY, et al. Psoriasis and the metabolic syndrome. Acta Derm Venereol. 2007;87:506-9.

11. Prey S, Paul C, Bronsard V, Puzenat E, Gourraud PA, Aractingi $\mathrm{S}$, et al. Assessment of risk of psoriatic arthritis in patients with plaque psoriasis: A systematic review of the literature. J Eur Acad Dermatol Venereol. 2010;24(Suppl.2):31-5.

12. Ho P, Barton A, Worthington J. Genetic epidemiology of psoriatic arthritis. Mod Rheumatol. 2004;14:91-100.

13. Gladman DD, Antoni C, Mease P, Clegg DO, Nash P. Psoriatic arthritis: Epidemiology, clinical features, course, and outcome. Ann Rheum Dis. 2005;64(Suppl.2):14-7.

14. Sánchez-Regaña M, Umbert P. Diagnosis and management of nail psoriasis. Actas Dermosifiliogr. 2008;99:34-43.

15. Mallbris L, Granath F, Hamsten A, Stahle M. Psoriasis is associated with lipid abnormalities at the onset of skin disease. J Am Acad Dermatol. 2006;54:614-21.

16. Lin TY, See LC, Shen YM, Liang CY, Chang HN, Lin YK. Quality of life in patients with psoriasis in northern Taiwan. Chang Gung Med J. 2011;34:186-96.

17. Schmitt J, Wozel G. The psoriasis area and severity index is the adequate criterion to define severity in chronic plaque-type psoriasis. Dermatology. 2005;210:194-9.

18. Neimann AL, Shin DB, Wang X, Margolis DJ, Troxel AB, Gelfand JM. Prevalence of cardiovascular risk factors in patients with psoriasis. J Am Acad Dermatol. 2006;55:829-35.

19. Nestle FO, Kaplan DH, Barker J. Psoriasis. N Engl J Med. 2009;361:496-509.

20. Mehta NN, Azfar RS, Shin DB, Neimann AL, Troxel AB, Gelfand JM. Patients with severe psoriasis are at increased risk of cardiovascular mortality: Cohort study using the General Practice Research Database. Eur Heart J. 2010;31:1000-6.

21. Ludwig RJ, Herzog C, Rostock A, Ochsendorf FR, Zollner TM, Thaci D, et al. Psoriasis: A possible risk factor for development of coronary artery calcification. Br J Dermatol. 2007;156:271-6.

22. Abuabara K, Azfar RS, Shin DB, Neimann AL, Troxel AB, Gelfand JM. Cause-specific mortality in patients with severe psoriasis: A population-based cohort study in the U.K. Br J Dermatol. 2010;163:586-92. 
23. Gisondi P, Tessari G, Conti A, Piaserico S, Schianchi S, Peserico $A$, et al. Prevalence of metabolic syndrome in patients with psoriasis: A hospital-based case-control study. Br J Dermatol. 2007;157:68-73.

24. Chen YJ, Wu CY, Shen JL, Chu SY, Chen CK, Chang YT, et al. Psoriasis independently associated with hyperleptinemia contributing to metabolic syndrome. Arch Dermatol. 2008;144:1571-5.

25. Alberti KG, Zimmet P, Shaw J. Metabolic syndrome - a new worldwide definition. A Consensus Statement from the International Diabetes Federation. Diabet Med. 2006;23:469-80.

26. Zimmet P, Magliano D, Matsuzawa Y, Alberti G, Shaw J. The metabolic syndrome: A global public health problem and a new definition. J Atheroscler Thromb. 2005;12:295-300.
27. Wakkee M, Thio HB, Prens EP, Sijbrands EJ, Neumann HA. Unfavorable cardiovascular risk profiles in untreated and treated psoriasis patients. Atherosclerosis. 2007;190:1-9.

28. Kimball AB, Jacobson C, Weiss S, Vreeland MG, Wu Y. The psychosocial burden of psoriasis. Am J Clin Dermatol. 2005;6:383-92.

29. Krueger GG, Feldman SR, Camisa C, Duvic M, Elder JT, Gottlieb $\mathrm{AB}$, et al. Two considerations for patients with psoriasis and their clinicians: What defines mild, moderate, and severe psoriasis? What constitutes a clinically significant improvement when treating psoriasis? J Am Acad Dermatol. 2000;43:281-5.

30. Choi J, Koo JY. Quality of life issues in psoriasis. J Am Acad Dermatol. 2003;49(Suppl.2):57-61. 